\title{
The origin and significance of spinal cord pulsation
}

\author{
H Matsuzaki $^{1}$, K Wakabayashi ${ }^{1}, \mathrm{~K}$ Ishihara ${ }^{1}, \mathrm{H}$ Ishikawa $^{1}, \mathrm{H}$ Kawabata ${ }^{2}$ and T Onomura ${ }^{2}$ \\ ${ }^{1}$ The Department of Orthopaedic Surgery, Nihon University School of Medicine; ${ }^{2}$ The Department of Orthopaedic \\ Surgery, Osaka Medical College, Nihon University, Surugadai Hospital, Tokyo 101, Japan
}

\begin{abstract}
We studied the origin and mechanism of spinal cord pulsation in ten dogs in order to elucidate its clinical significance. Under general anesthesia, a $6 \mathrm{~cm}$ length of the animals' cervical spinal cord was exposed and the cerebrospinal fluid removed. The amplitude of spinal pulsation was then measured by means of ultrasonography in Mode $\mathbf{M}$. The measurements were made after the spinal cord was cut: (1) on the cranial side; (2) on the cranial as well as caudal side, with the nerve roots and radicular arteries intact; (3) on both sides as in (2), which was then supplemented with the severance of the nerve roots and radicular arteries. It was demonstrated that, while the pulsation amplitude stood at an average of $88.0 \mu \mathrm{m}$ before the surgical treatment, it rose to $455.0 \mu \mathrm{m}$ in (1) and $274.8 \mu \mathrm{m}$ in (2), but dropped to nearly zero in (3). The 5.2-fold increase in pulsation following the cordotomy on the cranial side was attributed to two factors: (1) the increased spinal mobility due to the cordotomy; (2) the elevation of blood pressure. The results of the three different degrees of spinal detachment suggested that spinal pulsation derived mainly from the radicular arteries and that its presence indicated low tonicity in the spinal cord and favorable circulation in the radicular arteries.
\end{abstract}

Keywords: spinal cord; cerebrospinal fluid; circulation; spinal cord pulsation; ultrasonography

\section{Introduction}

The presence of spinal pulsation has been often used during operations as an index to estimate spinal decompression.

Nevertheless, its origin or mechanism has not yet been fully elucidated. Sufficient knowledge and accurate evaluation concerning these details are essential if spinal pulsation is to serve effectively as a spinal decompression index.

Various theories have been offered regarding the origin of spinal pulsation: as deriving from the radicular arteries; from the direct conduction of the changes in the pulsating brain tissue volume. The present experiments on dogs were intended to demonstrate the origin and mechanism of spinal pulsation and to evaluate its clinical significance.

\section{Materials and methods}

Ten hybrid adult dogs were intravenenously anesthetized with Nembutal and tracheally intubated, so that the animals were artificially ventilated by means of a respirator. After a laminectomy of the 3rd, 4th and 5th cervical vertebrae was carried out, the dura mater was exposed for some $6 \mathrm{~cm}$ and, with the surgical field filled with physiological saline the spinal pulsation was extradurally observed by ultrasonograms.

Subsequently, the dura mater was resected along

Correspondence: Hiromi Matsuzaki most of its circumference to expose the spinal cord. With the cerebrospinal fluid completely removed, measurements of the pulsation were carried out under three different experimental conditions that will be subsequently explained.

Spinal pulsation was studied by means of ultrasonography in Mode $\mathrm{M}(7.5 \mathrm{MHz})$, with its amplitude quantified. The Mode $\mathrm{M}$, a program commonly applied to the diagnosis of cardiac disease, can capture kinetic changes in a functioning organ as a series of images of its cross sections. In our present experiments, the difference in height at a certain point of the spinal cord, which resulted from the pulsation, was measured on ultrasonograms as the amplitude of spinal cord pulsation. The instrument used for the ultrasonography was Aloka's SSD-650CL (The Japanese company).

\section{(1) Experiment $A$}

In order to exclude the effects of the changing brain volume and central blood circulation, the exposed spinal cord was completely divided on its cranial side (Figure 1).

\section{(2) Experiment $B$}

Subsequent to Experiment $A$, the caudal side of the exposed spinal cord was also severed, so that both the central and the peripheral influences could be 
eliminated. Thus, the spinal cord was connected only to the nerve roots and their accompanying blood vessels (Figure 2).

\section{(3) Experiment $C$}

Finally, the remaining nerve roots and their accompanying blood vessels were cut away, leaving the spinal cord completely detached, and even free from the effects of the nerve roots as well as the radicular arteries and veins (Figure 3).

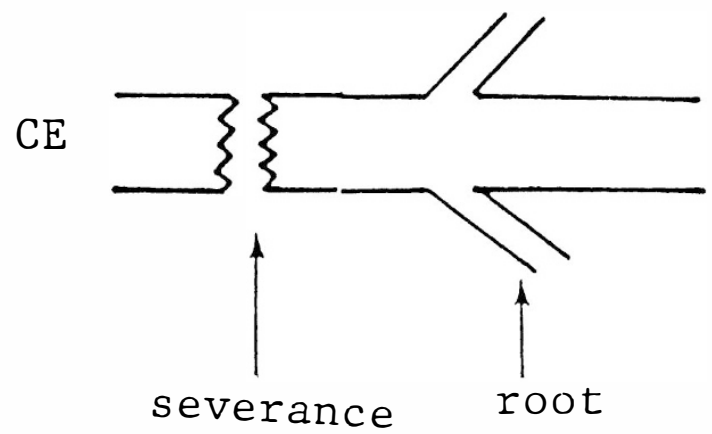

CA

Figure 1 Experiment A. Cordotomy (the arrow) on the cranial side, to exclude central influence. CE: Cranial Side, CA: Caudal Side

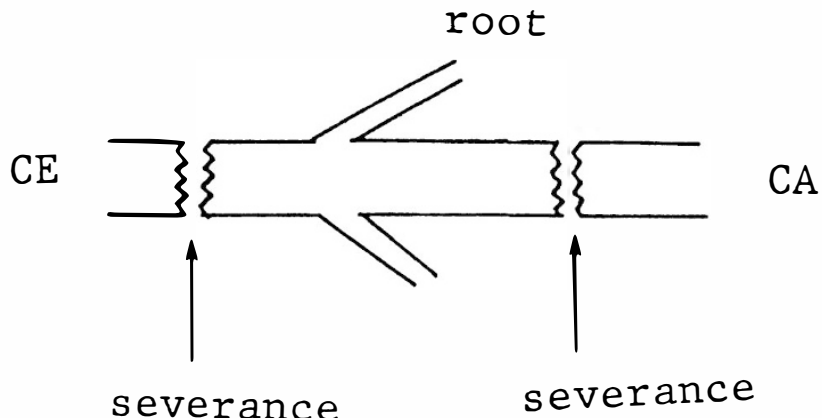

Figure 2 Experiment B. Spinal cord severed on both cranial and caudal sides (arrows), with only the nerve roots plus the radicular arteries and veins preserved

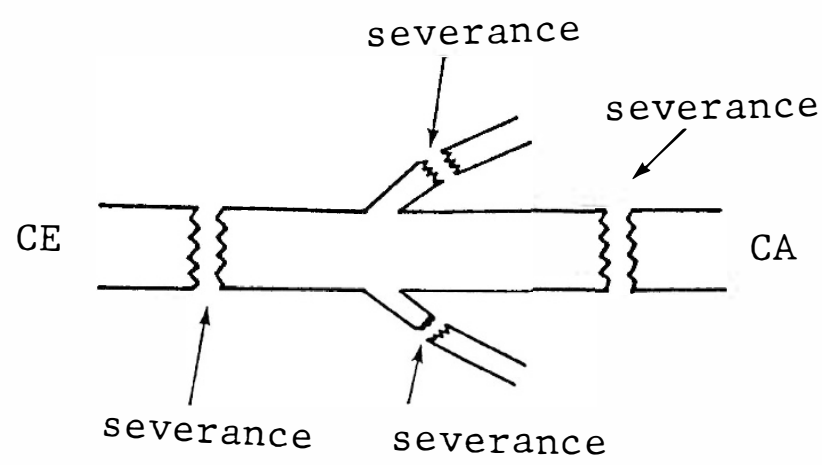

Figure 3 Experiment C. Spinal cord completely cut from the nerve roots and supporting blood vessels (arrows)
Under the foregoing three conditions, the amplitude of spinal pulsation was measured and evaluated. The pictures show an example of ultrasonographic pulsation measurement in Mode $M$ in Experiment $A$ (Figure 4). The one on the left gives the longitudinal view of a spinal cord: the arrow in the middle of the spinal cord indicates the site on whose cross section the pulsation is to be examined. The right-hand picture represents a series of images of the cross section in the pulsating spinal cord in Mode $\mathbf{M}$. The difference in height at this point, as indicated by the arrow, was measured and expressed in $\mu \mathrm{m}$ as the pulsation amplitude.

\section{Results}

As the amputated end tended to pulsate more vigoriously that did other parts of the cord, the amplitude was measured on the spinal stump where the pulsation was the greatest.

No spinal cord pulsation was observed in five of 10 dogs. Therefore, amplitudes of spinal cord pulsation in five dogs were analysed on this study.

\section{(1) Experiment $A$-free from the central influence}

Prior to the severance of the spinal cord on the cranial side, the pulsation amplitude ranged from a minimum of $9 \mu \mathrm{m}$ to a maximum of $310.3 \mu \mathrm{m}$ (mean: $88.0 \mu \mathrm{m}$ ). After the surgical procedure, the value rose to $27.2-$ $1068.9 \mu$ m (mean: $455.0 \mu \mathrm{m}$ ).

It was thus demonstrated that, apart from the wide divergence among individual subjects, a cordotomy on the cranial side incurred up to a 5.2 times increase in the pulsation amplitude, in comparison with the values before the procedure (Table 1).

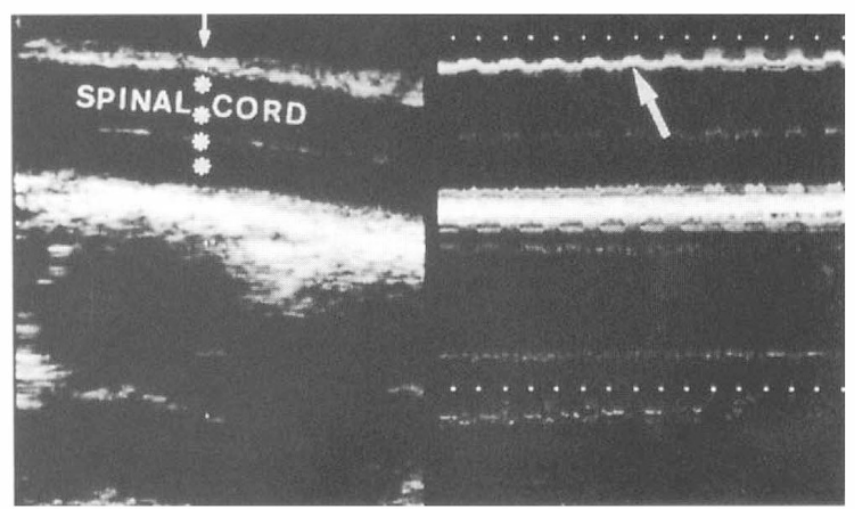

Figure 4 Left: Ultrasonogram of the spinal cord, longitudinal projection $(7.5 \mathrm{MHz})$; the movements of the spinal cross section at the indicated point (arrow) ultrasonographically depicted in Mode M. Right: M-mode of the pulsating spinal cord; pulsation amplitude determined by the measurement of the expanded spinal cord at the point indicated on the surface (arrow) 
Table 1 Amplitudes of Spinal Cord Pulsation

\begin{tabular}{lrrrr}
\hline & & \multicolumn{3}{c}{ Experiments $(\mu m)$} \\
Cases & Before severing $(\mu m)$ & $A$ & $B$ & $C$ \\
\hline 1 & 40.9 & 66.6 & 45.0 & 0 \\
2 & 9.0 & 27.2 & 20.0 & 0 \\
3 & 11.3 & 78.5 & 50.2 & 0 \\
4 & 310.3 & 1068.9 & 758.6 & 0 \\
5 & 68.9 & 1034.0 & 500.0 & 0 \\
Mean & 88.0 & 455.0 & 274.8 & 0 \\
SD & \pm 113.2 & \pm 487.4 & \pm 301.0 & \\
\hline
\end{tabular}
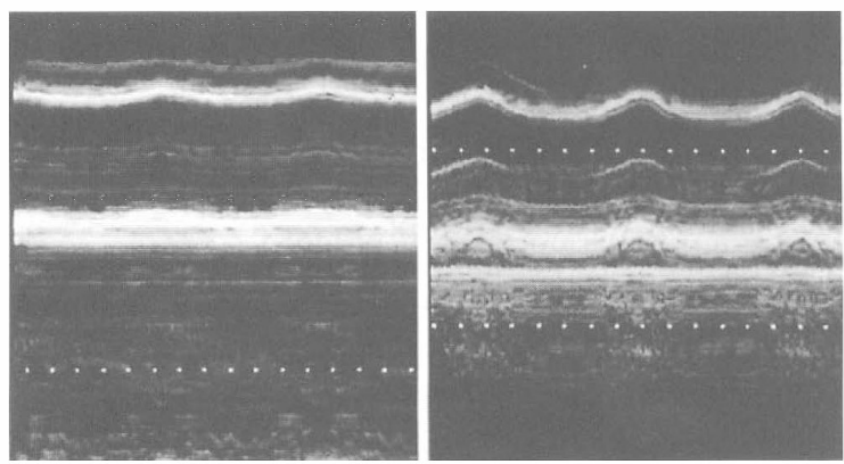

Figure 5 Left: Respiratory movements of the spinal cord smaller on the cranial side (amplitude: $45.9 \mu \mathrm{m}$ ). Right: Respiratory movements of the spinal cord the larger on the caudal side (amplitude: $113.6 \mu \mathrm{m}$ ). That is, closer to the thorax, the greater the respiratory influence

\section{(2) Experiment $B$-free from both central and peripheral} influences

When the spinal cord was cut at both ends while connected only to the nerve roots and accompanying blood vessels, the pulsation amplitude still remained in a high range between $20.0 \mu \mathrm{m}$ and $758.6 \mu \mathrm{m}$. The mean of $274.8 \mu \mathrm{m}$ represented a $40 \%$ decrease from that $455.0 \mu \mathrm{m}$ in Experiment A, suggesting that spinal pulsation was mainly caused by the radicular arteries coursing along the nerve roots (Table 1).

\section{(3) Experiment $C$-with the spinal cord completely detached}

Pulsation fell almost to zero, though there remained some spinal movement induced by respiration (Table 1).

\section{(4) The influence of respiration}

All the measurements in Experiment A, B and C were carried out with the animals in apnea. When pulsation was examined while they were artificially ventilated, it was found that both the influence of respiration and the increase in the amplitude tended to increase in proportion to the proximity of the site examined to the thorax or the caudal and of the exposed cervical spinal cord (Figure 5).

\section{Discussion}

Spinal pulsation was not always observed, which is also clinically experienced. It was completely absent in five of the ten animals. In these five, the blood pressure was not particularly hypotensive $(130 \mathrm{mmHg})$; changes in the position of the cervical vertebrae did not induce measurable pulsation either. We thus had to exclude these animals. In the remaining five, the pulsation amplitude varied considerably from case to case.

Spinal movements have two components: one is from cardiac pulsation and the other from respiration, though the latter cannot be called genuine pulsation. The results of the foregoing experiments indicated that spinal pulsation is derived mainly from the radicular arteries, rather than from the change in the brain volume or the influence of central and peripheral circulation. The result of Experiment $\mathrm{A}$ was quite interesting, as it showed a 5.2-fold increase in the pulsation amplitude following the cordotomy on the cranial side. The phenomenon might be explained in terms of two causative factors: (a) that the cordotomy decreased the tonicity and increased the mobility of the spinal cord; (b) that the cordotomy elevated blood pressure. As indicated by the results of the blood pressure measurements, which were carried out on four of the animals, a cordotomy of the cranial side tended to bring about a rise in blood pressure. When the blood pressure was raised with a hypertension from $100 \mathrm{mmHg}$ to $150 \mathrm{mmHg}$, the pulsation amplitude increased from $758.6 \mu \mathrm{m}$ to $896.5 \mu \mathrm{m}$, resulting in an $18 \%$ increase. Thus, the enhanced spinal pulsation following a cordotomy on the cranial side presumably resulted from the lowered tonicity in the spinal cord and the rise in blood pressure (Figure 6). In Experiment B, in which the spinal cord was amputated on the caudal side after Experiment A, the pulsation amplitude dropped from $45.05 \mu \mathrm{m}$ to $274.8 \mu \mathrm{m}$. This also suggested the influence exerted by the caudal portion of the cord.
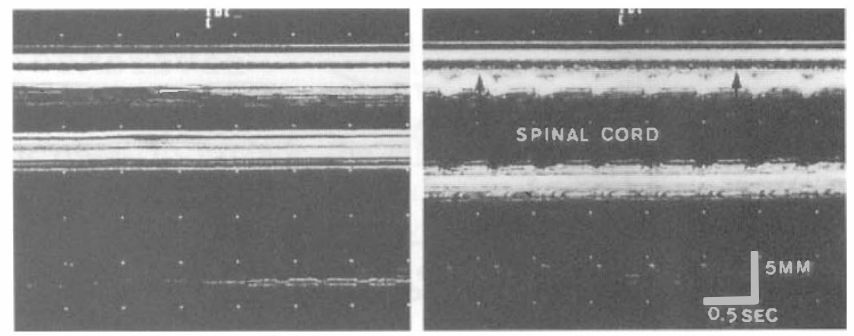

Figure 6 Left: Before the cordotomy on the cranial side in Experiment A; pulsation amplitude too weak and virtually not measured. Right: After the cordotomy; amplitude amounting to $1034 \mu \mathrm{m}$ (arrows) 
Cerebrospinal fluid moves back and forth $(10 \mathrm{~mm} /$ pulse) in the cervical canal. It is driven by the spinal and cerebral circulatory systems and does not appear to be exerting a direct influence on spinal pulsation. ${ }^{1-3}$ We confirmed this in the present experiments. After the cordotomies were made, we stuffed tampons at the cranial and caudal ends of the severed spinal canal, removing cerebrospinal fluid and replacing it with saline for ultrasonography. The subsequent findings of the enhanced spinal pulsation suggested that cerebrospinal fluid did not directly affect the pulsation.

Suspending the spinal cord in its fluidity, cerebrospinal fluid certainly provides an environment quite favorable for spinal movement. Driven by the expanding and contracting thorax in respiration, it mediates the respiratory movements of the spinal cord, which increase as the spinal cord approaches the thorax. Nevertheless, this respiratory component is not regarded as genuine spinal pulsation.

The energy for spinal pulsation appears to originate from the blood flow in the radicular arteries. They run along the nerve roots and turn nearly at a right angle to from the anterior and posterior spinal arteries. Colliding against the arterial wall at the curve, the pulsating blood stream displaces the nerve roots back and forth (Figure 7). This in turn leads the spinal cord to vibrate which is reinforced by the throbbing arteries within the spinal canal. In an environment that allows high mobility, the entire spinal cord thus pulsates as if it were a blood vessel. ${ }^{4}$

We were able to analyse clinically observed spinal movements into two patterns. One consists of the movements of the entire spinal cord as the result of the extraspinal cause, including the pulsating radicular arteries as well as the central and peripheral influences beyond the cordotomized sites. All of this is further

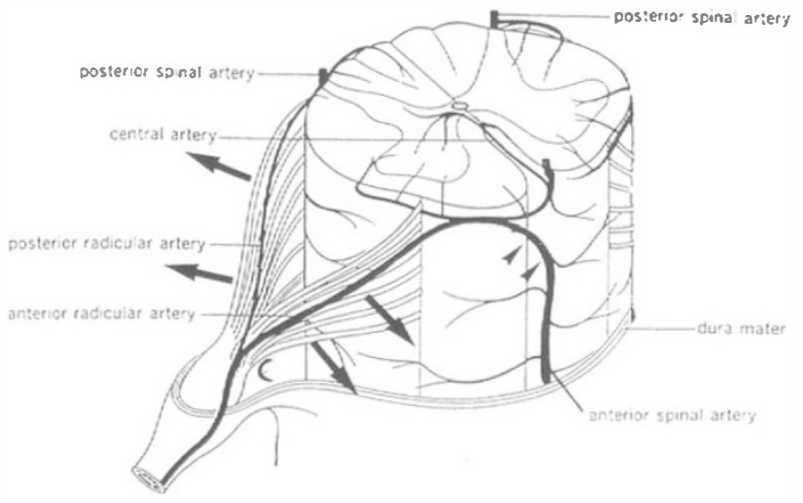

Figure 7 Mechanism for spinal pulsation as based on the role of the radicular arteries. The blood stream in the radicular arteries clashes against the vascular walls at the curves where the radicular arteries become the anterior and posterior spinal arteries (arrow heads). The impact pushes the nerve roots to and fro, moving the spinal cord back and forth (arrows)

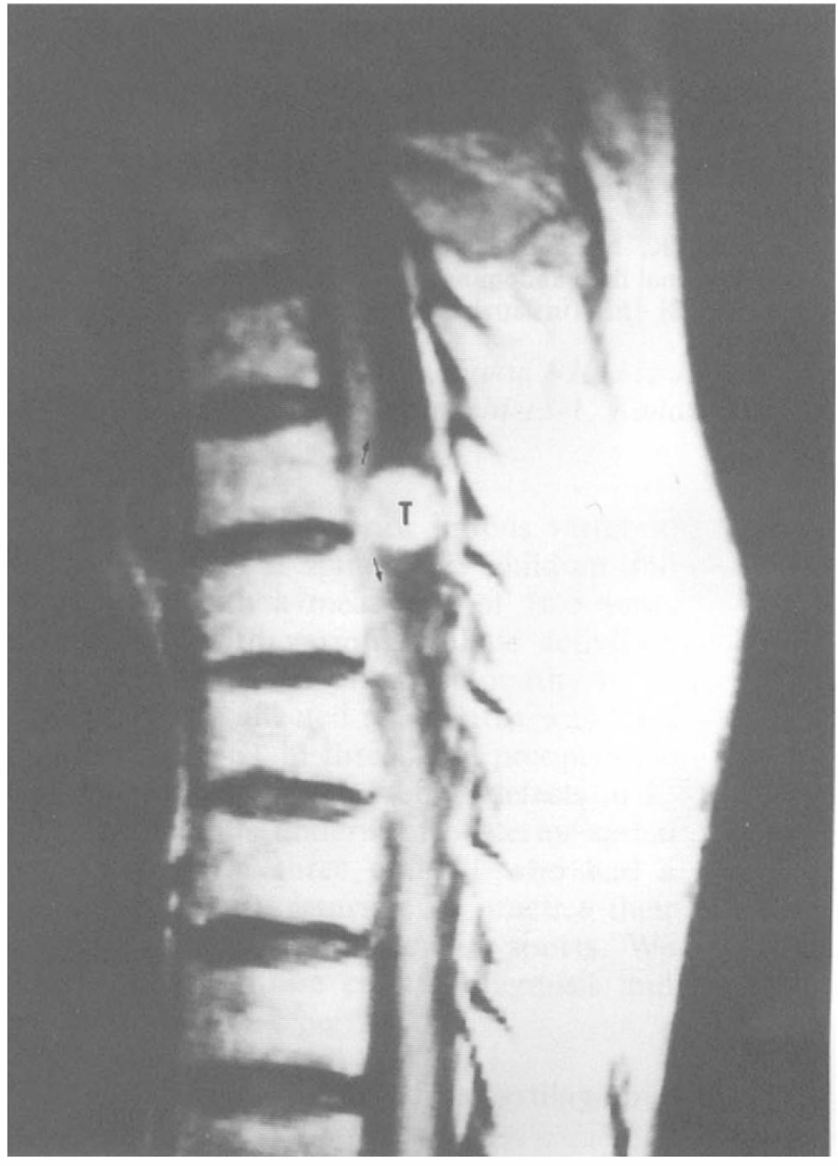

Figure 8 MRI of a spinal cord with a tumor. The tumor compresses and stretches the spinal cord (arrow), reducing its flexibility. T: tumor

complicated by the pulsation of the intraspinal arteries. The other patter originated from respiration, adding further complexity to the spinal pulsation. In the presence of a spinal tumor, the incision of the dura matter often fails to restore spinal pulsation. This may be due to the fact that the spinal cord has become tonic from the tumour's pressure and has lost its flexibility (Figure 8).

\section{Conclusions}

Spinal cord pulsation originates mainly from the radicular arteries. It is facilitated in the absence of spinal tonicity and enhanced by a rise in blood pressure. Clinically, its presence implies satisfactory circulation in the radicular arteries and the absence of tonicity in the spinal cord. In our future investigations we must elucidate the influence of the caudal portion of the spinal cord by sectioning this part first. Yet further elaboration of the experimental methodology appeared necessary because of the difficulty of material procurement and the frequent absence of pulsation. 


\section{References}

1 Jokich PM, Rubin JM, Dohrmann GJ. Intraoperative ultrasonic evaluation of spinal cord motion. J Neurosurg 1984; 60: $707-711$.

2 Tsukamoto $\mathrm{H}$, Inada $\mathrm{Y}$. Artifacts of spinal MRI: The clinical significance artifacts. Spine \& spinal cord 1992; 5: 829 - 836 (in Japanese).

3 Urayama K, Nakamura K, Nagashima K. Ooi Y. Lumbar cerebrospinal fluid abdominal aortic surgery. Spine \& spinal cord 1992; 6: 521 - 527 (in Japanese).

4 Hamer J, Alberti E, Wiedemann K. Influence of systemic and cerebral vascular factors on the cerebrospinal fluid pulse waves. $J$ Neurosurg 1977; 46: $36-45$. 